\title{
Functional advantage of NPC-related V-val subtype of Epstein-Barr virus nuclear antigen 1 compared with prototype in epithelial cell line
}

\author{
SHI-JUAN MAI ${ }^{1}$, TADAMASA OOKA ${ }^{2}$, DA-JIANG LI ${ }^{1}$, MU-SHENG ZENG ${ }^{1}$, RI-CHENG JIANG ${ }^{1}$, \\ XING-JUAN YU ${ }^{1}$, RU-HUA ZHANG ${ }^{1}$, SHI-PING CHEN ${ }^{1}$ and YI-XIN ZENG ${ }^{1}$
}

\begin{abstract}
${ }^{1}$ Department of Experimental Research, State Key Laboratory of Oncology in Southern China, Cancer Center, Sun Yat-sen University, 651 DongFeng Road East, GuangZhou 510060, P.R. China; ${ }^{2}$ Laboratoire de Virologie Moléculaire, UMR5537, CNRS, Faculté de Médecine R.T.H. Laennec, Université Lyon-1, 69372 Lyon, Cedex 08, France
\end{abstract}

Received August 11, 2006; Accepted September 18, 2006

\begin{abstract}
Epstein-Barr virus (EBV) is closely associated with nasopharyngeal carcinoma (NPC) and the viral nuclear antigen 1 (EBNA1) plays a crucial role in viral latency. Three EBNA1 subtypes, P-ala, V-thr and V-val have been detected from healthy carriers in Guangzhou area. A close relation of V-val EBNA1 with NPC was suggested by its preference to infect NPC cells. We therefore investigated the functional difference among these three EBNA1 subtypes in human epithelial cell line. The three coding sequences of the EBNA1 subtypes were cloned into the pGFP-C2 vector, and transfected into 293 cells, respectively. Effect of EBNA1 expression on cell proliferation was examined. The maintenance activity and expression level of EBNA1-plasmid in 293 cells were evaluated by using GFP as a reporter. The expression of P-ala, V-thr or V-val EBNA1 had no effect on 293 cell growth, while the relative average intensity of fluorescence after 14-day selection in V-val-EBNA1/293 cells was statistically higher than P-ala-EBNA1/293 $(\mathrm{P}<0.05$, $\mathrm{t}$ test). We suggest that V-val EBNA1 with the functional advantage compared with prototype shown in this study might contribute to the tumorigenesis of NPC by increasing the expression of itself or other viral or cellular genes.
\end{abstract}

\section{Introduction}

Epstein-Barr virus (EBV) infects most of the global population, but only a small percentage of infected individuals develop EBV-associated malignancies including nasopharyn-

Correspondence to: Dr Yi-Xin Zeng, Department of Experimental Research, Cancer Center, Sun Yat-sen University, 651 Dong-Feng Road East, Guangzhou 510060, P.R. China

E-mail: yxzeng@gzsums.edu.cn

Key words: Epstein-Barr virus, EBNA1, nasopharyngeal carcinoma, variant geal carcinoma (NPC), Burkitt's lymphoma, Hodgkin's disease and various T-cell lymphomas (1). Since NPC occurs with high frequency in Asian populations, especially among people of Cantonese ancestry, both the genetic background and the EBV variation have been suggested to influence the carcinogenesis of NPC. We previously localized an NPC susceptibility locus to chromosome 4p12-p15 by an entire genomic scan linkage analysis in families at high-risk of NPC from Guangdong Province (2). On the other hand, we identified a unique EBV strain GD1 (GenBank accession no. AY961628) that importantly differs from the B95.8 prototype is widespread in Guangdong (3).

EBV encoded nuclear antigen 1 (EBNA1) is the only latent protein consistently expressed in viral-associated tumors and crucial for viral latent infection in DNA replication, transcription and partitioning of the viral genomes during cell division by binding to viral latent replication origin region, oriP (4). The predicted amino acid sequence of EBNA1, which consists of 641 aa, can be separated into unique $\mathrm{N}$ - and $\mathrm{COOH}$-terminal domains joined by internal glycine/alaninerich repeat (GAR) sequences (90-327 aa). Two domains (domain A and B) within amino terminus of EBNA1 (33-89 and 328-378 aa) have AT hook activity that strongly correlates with the ability of EBNA1 to associate with metaphase chromosomes and to support the replication and partitioning of oriP plasmids (5). EBNA1 residues that mediate DNA binding and dimerization have been localized to the $\mathrm{C}$-terminal portion of the protein between amino acids 459 and 607 (6). The crystal structure of this region reveals two structural motifs, a core domain (504-604 aa), which mediates protein dimerization and a flanking domain (461-503 aa), which mediate the base contacts with DNA (7). No profound effect on the function of EBNA1 was found in the GAR domain, while it has been shown to interfere with antigen processing and/or presentation of EBNA1 to the cellular immune compartment (8).

Based on the variation at 487 aa, EBNA1 was classified into P-ala (P, prototype), P-thr, V-val (V, variant), V-leu and V-pro subtypes (9). EBNA1 is referred to as P-ala when alanine occurs at amino acid 487 , which represents the sequence of prototype B95.8. P-thr indicates threonine at amino acid 487 , which closely relates to P-ala. V-val contains 
multiple variations of amino acids at other sites besides valine at amino acid 487. Likewise, V-leu or V-pro indicates that leucine or proline occurs at amino acid 487, respectively, which also contains multiple variations at other sites as compared with B95.8 (9-11).

In our previous study, three subtypes of EBNA1, P-ala, $\mathrm{V}$-thr and $\mathrm{V}$-val, were detected in peripheral blood lymphocytes (PBL) of 144 cases of EBV healthy carriers from Guangzhou area in Southern China (12). V-thr, which contains 17 amino acid mutations compared with $\mathrm{P}$-ala is different from P-thr subtype described by Bhatia which is similar to P-ala. EBV in healthy carriers in Guangzhou area encompasses multiple EBNA1 subtypes in peripheral blood, among them $\mathrm{V}$-val and V-thr were dominant subtypes, while V-thr was absent in NPC tissues. The close relation between V-val EBNA1 and NPC has been suggested. V-val was the only subtype of EBNA1 in 71 cases of Chinese NPC biopsies $(10,13,14)$. In another report, 91.67\% (33/36) of the EBNA1 subtypes from NPC specimens were single V-val subtypes, and the remaining three patients were coinfected with $\mathrm{V}$-val and P-ala (12). In addition, the variations of V-val derived from biopsies of NPC were identical to those derived from PBL and throat washings in the context of $\mathrm{N}$-terminus and $\mathrm{C}$-terminus of EBNA1 $(12,13)$. These facts indicated that substrain of EBV with V-val subtype of EBNA1 infects NPC preferentially and this preferential selection is unlikely ascribable to the variations of EBNA1 itself or the heritable susceptibility of the host. Therefore, it is reasonable to infer some advantage with V-val EBNA1 over the other subtypes for the development of NPC. In this study, the difference of biological functions among EBNA1 subtypes, P-ala, V-thr and V-val on epithelial cell line were detected and compared.

\section{Materials and methods}

Materials. The B95.8 cell and human embryonic kidney epithelial cell line 293 was cultured in RPMI-1640 containing $10 \% \mathrm{FBS}$ and incubated at $37^{\circ} \mathrm{C}$ in an incubator with $5 \% \mathrm{CO}_{2}$. NPC biopsies were collected from Cancer center, Sun Yat-sen University.

Construction of recombinant plasmids. Prototype EBNA1 (P-ala) derived from B95.8 cells, V-thr subtype from PBL of healthy carrier, and V-val from NPC biopsy were amplified using the primer introduced HindIII and BamHI restriction sites (S: 5'-aagcttgtgaatcatgtctgacg-3', 107, 942-107, 959 nt; A: 5'-ggatccagttacatcactcctg-3', 109, 882-109, 867 nt). PCR products were cloned into pGEM-T easy vector (Promega) and subcloned into pGFP-C2 vector. Pyrobest DNA polymerase (Takara Biotechnology Co., Ltd.) with proofreading function was used. Recombinants were confirmed by enzymes digestion and sequencing.

Gene transfection. pGFP, P-ala-EBNA1-pGFP, V-thrEBNA1-pGFP and V-val-EBNA1-pGFP plasmids were transfected into 293 cells separately by using the Lipofectamine 2000 (Invitrogen) according to the manufacturer's protocol. EBNA1 stably expressing cells used in cell growth experiment were obtained after 4-week selection under G418 (400 $\mu \mathrm{g}$ / $\mathrm{ml}$ ) and confirmed by RT-PCR and Western blot assay.
Cell growth. The stably transfected 293 cells were seeded at a density of $1 \times 10^{4}$ cells/well in 6-well plates. The number of cells at various time-points was determined by cell counting. The viability of cells was determined by trypan blue exclusion. Cell doubling (CD) was calculated by using the formula: $\lg \left(\mathrm{N}_{\mathrm{t}} /\right.$ $\mathrm{N}_{0}$ )/lg2, where $\mathrm{N}_{\mathrm{t}}$ or $\mathrm{N}_{0}$ is the cell numbers at different timepoint $T_{t}$ or $T_{0}$. Doubling time was obtained by dividing the time interval $\left(\mathrm{T}_{\mathrm{t}}-\mathrm{T}_{0}\right)$ by CD. Flow cytometry (FCM) (BeckmanElite) analysis of DNA content was performed to assess the cell cycle phase distribution according to the procedure described before (15). The populations of cells in the G1, G2 and $\mathrm{S}$ phases were determined by FCM, and results analyzed using Multicycle software. Statistical analysis was carried out by means of double-sided t-test. Each experiment was performed at least three times independently.

Maintenance and expression of EBNA1-plasmids in 293 cells. The maintenance activity and expression level of EBNA1plasmids were tested by the index of the percent of GFPexpressing cells and the average intensity of green fluorescence in GFP-expressing cells by flow cytometer (BeckmanElite). The 293 cells were plated in 6 -well plates at $1 \times 10^{6}$ cells/well and, and transfected with $3 \mu \mathrm{g}$ of pGFP plasmids expressing P-ala, V-thr, V-val EBNA1 or no EBNA1. Cell lines were re-suspended at 18 -h post-transfection, and half of the cells in each well were harvested and detected for GFPexpressing cell percent and average intensity of green fluorescence in GFP-expressing cells on FCM to normalize transfection efficiency. The other half of cells were re-plated and grew under G418 $(400 \mu \mathrm{g} / \mathrm{ml})$ selection. The selective medium was changed every 3 days for 2 weeks without trypsinization since a mass of cells initially died from antibiotics, after which the cells were collected and detected for GFP-expressing cell percent and average intensity of green fluorescence in GFP-expressing cells on FCM. Experiments were performed in three replicate wells for each cell line.

\section{Results}

Sequencing variations. The sequence of prototype EBNA1 is identical to B95.8 sequence in NCBI (GeneID: 59074). The nucleotide mutations in $\mathrm{V}$-thr and $\mathrm{V}$-val variants were the same as in our previous report (12) and V-val is also identical with EBV strain GD1 (GeneID: 84519641). The amino acid mutations of V-thr and V-val subtypes compared with P-ala was shown in Table I. The variations of V-val EBNA1 lead to 5 aa mutations in $\mathrm{N}$ terminal and 11 mutations in $\mathrm{C}$ terminal of the protein, most of which were located in apparently functionally significant regions according to a detailed mutational analysis of the functional domains of B95.8 EBNA1 (6). Three aa mutations in the flanking domain of EBNA1 might affect the DNA-binding affinity, in which a potential important change in position 487 from an alanine residue in the B95.8 EBNA1 to a valine residue in the v-val EBNA1 since this residue is located in an important region for sequence-specific DNA recognition (459-487 aa) (16). Four aa mutations fall within the core domain thought to be involved in EBNA1 dimerization. In the AT hook containing domains that bind the scaffold-associated regions on metaphase chromosomes, two amino acid mutations were 
Table I. The amino acid changes observed in the EBNA1 gene coding sequence of the V-thr and V-val subtypes compared with P-ala.

\begin{tabular}{|c|c|c|c|c|c|c|c|c|c|c|c|c|c|c|}
\hline \multirow{2}{*}{$\begin{array}{l}\text { EBNA1 } \\
\text { subtypes }\end{array}$} & \multicolumn{14}{|c|}{ No. of amino acid } \\
\hline & 16 & 18 & 20 & 24 & 27 & 85 & 91 & 357 & 364 & 369 & 411 & 418 & 429 & 439 \\
\hline & & & & & & \multicolumn{5}{|c|}{ AT hook } & & & & \\
\hline P-ala & Glu & Gly & Thr & Glu & Gly & Thr & Ala & Gly & Gly & Ala & Glu & His & Val & Ala \\
\hline V-thr & Gln & Glu & & Asp & Ser & Ser* & & Glu $^{*}$ & & Asp* & & & Met & \\
\hline \multirow[t]{3}{*}{ V-val } & Gln & Glu & Ser & & & Ser* & Thr & & Arg* & & Asn & Leu & & Thr \\
\hline & 476 & 487 & 492 & 499 & 502 & 524 & 528 & 533 & 563 & 574 & 585 & 594 & 595 & \\
\hline & \multicolumn{5}{|c|}{ Flanking domain } & \multicolumn{8}{|c|}{ Core domain } & \\
\hline P-ala & Pro & Ala & Ser & Asn & Thr & Thr & Ile & Leu & Met & Val & Thr & $\operatorname{Arg}$ & Val & \\
\hline V-thr & $\mathrm{Gln}^{*}$ & Thr ${ }^{*}$ & Cys* & & & $\mathrm{Ile}^{*}$ & & & $\mathrm{Ile}^{*}$ & Gly* & Pro* & Lys* & $\mathrm{Ala}^{*}$ & \\
\hline V-val & & $\mathrm{Val}^{*}$ & & $\mathrm{Glu}^{*}$ & Asp* & $\mathrm{Ile}^{*}$ & Val $^{*}$ & $\mathrm{Ile}^{*}$ & & & & Lys* $^{*}$ & & \\
\hline
\end{tabular}

${ }^{*}$ The mutated amino acids that located in functional regions.

A.

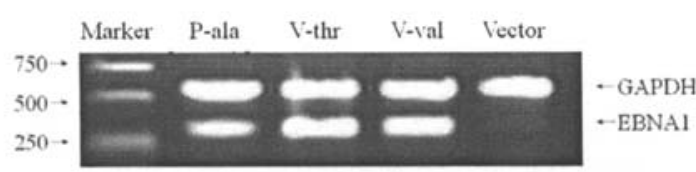

B.

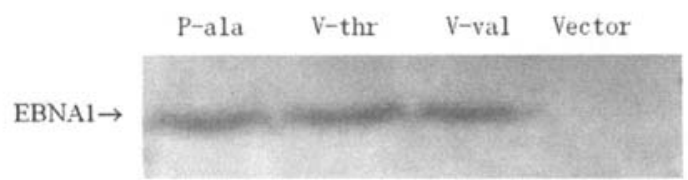

Figure 1. Confirmation of EBNA1 expression by RT-PCR and Western blot assay. (A), Total-RNA was isolated from transfected cells using Trizol reagent. The product of the reverse transcriptase reaction was used for amplication of a 329-bp fragment in EBNA1 gene C-terminal using primers 5'-tgcaaagggacccacggtggaacag-3' (upstream, 109, 260-109, $241 \mathrm{nt}$ ) and 5'-tca aaataataggcttcccctacag-3' (downstream, 109, 549-109, 569 nt) and GAPDH cDNA (580 bp) served as an internal control. (B), EBNA1 protein expression was detected with $40-\mu \mathrm{g}$ protein extracted from each cell line at 72 -h posttransfection by Western blot assay using EBNA1 monoclonal antibody vC-20 (Santa Cruz).

detected (85 aa: $\mathrm{T} \rightarrow \mathrm{S}$ and 364 aa: $\mathrm{G} \rightarrow \mathrm{R}$ ) in domain $\mathrm{A}$ and $\mathrm{B}$ in $\mathrm{V}$-val subtype. Five aa mutations in $\mathrm{N}$ terminal and 12 mutations in $\mathrm{C}$ terminal were detected in V-thr EBNA1, and most of them were different from the mutations in $\mathrm{V}$-val subtype.

Cell proliferation. EBNA1 expression in 293 cells after longterm selection of G418 was confirmed by RT-PCT and Western blot assay (Fig. 1). All the cell lines were observed to keep their morphology and characteristics during long-term culture. It was shown in the cell growth curve (Fig. 2) that all the cell

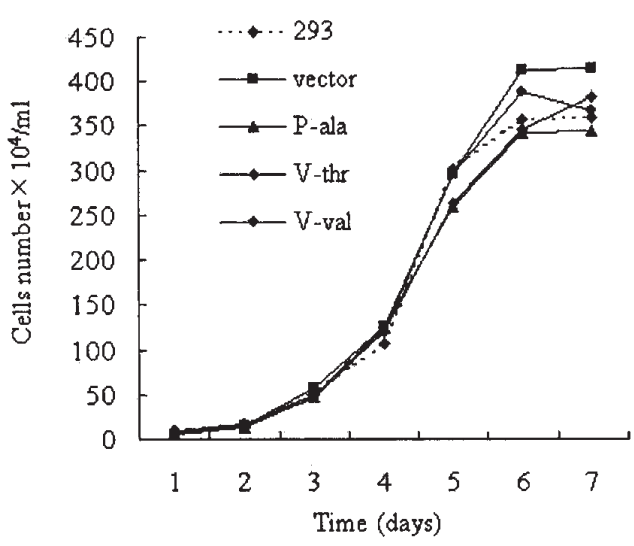

Figure 2. Curve of cell growth. Cell growth was not affected by P-ala, V-thr or V-val EBNA1 expression. The number of cells at various time-points was determined by cell counting. The viability of cells was determined by trypan blue exclusion. Each experiment was performed at least three times independently.

lines grow in logarithmic phase from the fourth to the fifth day, and enter the flat phase in the next two days. There was no significant difference in cell doubling time among 293 (23.26 $\pm 6.12 \mathrm{~h})$, vector/293 (18.97 $\pm 3.06 \mathrm{~h})$, P-ala-EBNA1/ $293(19.27 \pm 3.21 \mathrm{~h}), \mathrm{V}-$ thr-EBNA1/293 $(21.44 \pm 2.97 \mathrm{~h})$ and V-val-EBNA1/293 $(22.95 \pm 3.72 \mathrm{~h})$ cells $(\mathrm{P}>0.05$, t-test $)$. The cell cycle distribution was also not affected by EBNA1 expression by FCM detection (Table II).

Maintenance and expression of EBNA1-plasmids. The relative percent of GFP-expressing cells and the average intensity of green fluorescence in GFP-expressing cells detected by flow cytometer were calculated by dividing the value measured after 14-days selection under G418 by the value detected at 
Table II. Cell doubling time and cycle phase distribution.

\begin{tabular}{|c|c|c|c|c|c|}
\hline Cell lines & DT (h) & G1 & $\mathrm{G} 2$ & $\mathrm{~S}$ & $\mathrm{G} 2 / \mathrm{G} 1$ \\
\hline 293 & $23.26 \pm 6.12$ & 49.6 & 11.1 & 39.3 & 2.107 \\
\hline Vector/293 & $18.97 \pm 3.06$ & 49.1 & 13.7 & 37.1 & 2.087 \\
\hline P-ala-EBNA1/293 & $19.27 \pm 3.21$ & 48.4 & 14.7 & 37.0 & 1.996 \\
\hline V-thr-EBNA1/293 & $21.44 \pm 2.97$ & 44.6 & 16.8 & 38.6 & 1.938 \\
\hline V-val-EBNA1/293 & $22.95 \pm 3.72$ & 48.0 & 14.2 & 37.8 & 1.972 \\
\hline
\end{tabular}

Cell doubling time and cycle phase distribution were not affected by P-ala, V-thr or V-val EBNA1 expression compared with untransfected or vector transfected 293 cells according to the statistical analysis (t-test). DT, doubling time.

Table III. Maintenance and expression of EBNA1-plasmids in long-term selected transfectants.

\begin{tabular}{lccccccccc}
\hline & \multicolumn{2}{c}{$\begin{array}{c}\text { Average percent of } \\
\text { GFP-expressing } \\
\text { cells (\%) }\end{array}$} & & \multicolumn{2}{c}{$\begin{array}{c}\text { Average intensity } \\
\text { of fluorescence }\end{array}$} \\
\cline { 2 - 3 } Plasmids & $18 \mathrm{~h}$ & $14 \mathrm{~d}^{\mathrm{b}}$ & Ratio $^{\mathrm{a}}$ & & $18 \mathrm{~h}$ & $14 \mathrm{~d}^{\mathrm{b}}$ & Ratio $^{\mathrm{a}}$ \\
\hline P-ala-EBNA1 & 31.19 & 65.10 & 2.09 & & 14.40 & 6.92 & 0.48 \\
V-thr-EBNA1 & 33.80 & 60.81 & 1.80 & & 10.81 & 6.12 & 0.57 \\
V-val-EBNA1 & 32.02 & 63.93 & 2.00 & & 14.21 & 8.86 & 0.62 \\
\hline
\end{tabular}

${ }^{a}$ The ratio was calculated by dividing the value detected after 14-day culture by the one of 18 -h post-transfection. Enhanced relative fluorescence intensity was found in V-val-EBNA1/293 cells compared with P-ala-EBNA1/293 cells after 14-day selection $(\mathrm{P}<0.05, \mathrm{t}$-test $)$. ${ }^{b}$ d, days.

18-h post-transfection to normalize transfection efficiency. The possible difference of GFP-expressing cell percent and fluorescence intensity among EBNA1 transfected cell lines was evaluated by the t-test. The GFP-expressing cell percent increased nearly two fold after 14-day selection compared to 18 -h post-transfection in all cell lines, while there was no obvious difference in the ratio of GFP-expressing cell percent in P-ala (2.09), V-thr (1.80) and V-val (2.00) EBNA1 transfected cells $(\mathrm{P}>0.05, \mathrm{t}$-test). The average intensity of fluorescence decreased after selection in all cell lines, and the ratio of fluorescence intensity after 14-day selection compared to that at 18 -h post-transfection was gradually higher from P-ala-EBNA1 (0.48), V-thr-EBNA1 (0.57) to Vval-EBNA1 (0.62) transfected cells. There is a significant difference between $\mathrm{V}$-val and $\mathrm{P}$-ala $(\mathrm{P}<0.05$, t-test $)$ transfected cells (Table III).

\section{Discussion}

EBNA1 is consistently expressed in all EBV-associated tumors and essential for the persistence and replication of the
EBV genome in latently infected cells. Although two reports $(17,18)$ demonstrated a direct involvement of EBNA1 in the oncogenic process, most studies are consistent with EBNA1's having no effect (or a small effect) on cell growth or survival $(19,20)$. However, the function of EBNA1 variants other than prototype have not been previously studied in detail. Recent studies suggest that certain EBNA1 variants show selective geographical tropism and are preferentially associated with different EBV-positive malignancies; for example, $\mathrm{V}$-val is the dominant subtype in Asian regions and appear to be associated with Asian NPC patients but not found in Danish NPC biopsies (21), V-leu is present in Burkitt's lymphoma, and EBV with P-thr EBNA1 were the predominant virus in general population in America and Africa (9). The key role of EBNA1 in EBV biology has led to speculation that tumor-specific EBNA1 variants may account for the observed variations in the incidence of EBV-associated tumors.

It was shown in our previous study that the distribution characteristics of EBNA1 subtypes were different among PBL of EBV healthy carriers, PBL of NPC patients and NPC biopsies in Guangzhou area. Healthy carriers $(77.08 \%)$ were infected by 2 or 3 EBNA1 subtypes, whereas $97.22 \%$ of the PBL of NPC patients and $91.67 \%$ of NPC biopsies revealed only one EBNA1 subtype. The detection rate of P-ala, V-thr and V-val subtype of EBNA1 were $17.36 \%$ (25/144), $64.58 \%$ $(93 / 144)$ and $98.61 \%(142 / 144)$ respectively in PBL of healthy carriers, and were $11.11 \%$ (4/36), 19.44\% (7/36) and $72.22 \%(26 / 36)$ respectively in PBL of NPC patients, and were $8.33 \%(3 / 36), 0 \%(0 / 36)$ and $91.67 \%$ (33/36) respectively in NPC tissues (12). It was shown that EBV with V-val EBNA1 infects NPC tissue preferentially. In the present study, the biological functions of three EBNA1 subtypes were compared by introducing P-ala, V-thr and V-val EBNA1 into 293 cells, respectively.

No effect on cell growth rate or cell cycle distribution was found in either prototype or variants of EBNA1 transfected 293 cells, which suggest that EBNA1 subtypes do not have a direct transforming activity. Since the crucial function of EBNA1 is to enable persistence and enhance transcription from EBV episomes, we compared the maintenance and expression of GFP plasmid in EBNA1 transfected 293 cells. The GFP-expressing cell percent and the relative average intensity of fluorescence in GFP-expressing cells were 
measured at the same time by FCM. After 14-day culture under selection, no obvious difference of GFP-expressing cell percent was found among the three subtypes, while the relative average intensity of fluorescence in GFP-expressing cells was significantly higher in V-val-EBNA1 transfected cells than in P-ala transfectants. This difference is not likely due to the viability of transfectants since there is no difference of cell doubling time among these cell lines. The transfection efficiency for each cell line has no obvious difference at 18-h post-transfection, and the EBNA1 protein expression level that was showed in Western blot assay at 3 days post-transfection are also similar among the three subtypes (Fig. 1B). Since the percent of GFP-expressing cells has no difference between P-ala and V-val transfected cells, the maintenance and partition ability of the plasmid should be similar between these two cell lines. The increased intensity of fluorescence indicate the expression of $\mathrm{V}$-valEBNA1-GFP plasmid might be enhanced compared with Pala-EBNA1-GFP plasmid after long-term culture. It is known that EBV cannot be detected from normal nasopharyngeal mucosa. This enhancement of V-val-EBNA1 might be significant and necessary in initiation of persistent latent infection of EBV in nasopharyngeal epithelial cells in vivo considering the crucial role of EBNA1 in EBV latency. However, the mechanism of this enhanced activity of EBNA1 is unclear since we have not introduced EBNA1binding sequence orip into the system. Our finding indicate a direct role of EBNA1 in maintenance and transactivation function and further study is necessary to prove it.

V-thr-EBNA1 was also frequently detected in healthy carriers in Guangzhou, whereas no V-thr was detected from 87 NPC biopsies in our previous study $(12,13)$. This finding indicate a functional difference between $\mathrm{V}$-thr and $\mathrm{V}$-val EBNA1. In this study, the relative average intensity of GFP fluorescence after 14-day selection in V-thr-EBNA1 transfected cells was lower than that of V-val and higher than P-ala, although no significant difference was found. It is possible that the difference of $\mathrm{V}$-thr with the other subtypes might be more obvious after a longer period of culture or with a more sensitive method.

EBNA1 acts as a transcriptional activator and enhances the expression of several viral genes including the EBNAs and LMP1 from the viral Cp-promoter (22). EBNA1 is also associated with increased expression levels of selected cellular genes including CD25, bcl-xL and V(D)J recombinaseactivating genes RAG1 and -2 (23-26). These proteins might contribute to genetic instability and cellular transformation. Our findings in this study show enhanced plasmid expression for V-val EBNA1 transfected cells. We suggest a potential contribution of NPC related EBNA1 subtype, V-val, to EBV associated tumorigenicity by increasing the expression of itself or other viral or cellular genes.

\section{Acknowledgements}

This study was supported by the National Research Projects in Science and Technology (No. 2002BA711A03) and the National Natural Science Foundation of P.R. China (No. 30400493).

\section{References}

1. Griffin BE: Epstein-Barr virus EBV and human disease: facts, opinions and problems. Mutation Res 462: 395-405, 2000.

2. Feng BJ, Huang W, Shugart YY, Lee MK, Zhang F, Xia JC, Wang HY, Huang TB, Jian SW, Huang P, Feng QS, Huang LX, Yu XJ, Li D, Chen LZ, Jia WH, Fang Y, Huang HM, Zhu JL, Liu XM, Zhao Y, Liu WQ, Deng MQ, Hu WH, Wu SX, Mo HY, Hong MF, King MC, Chen Z and Zeng YX: Genome-wide scan for familial nasopharyngeal carcinoma reveals evidence of linkage to chromosome 4. Nat Genet 31: 395-399, 2002.

3. Zeng MS, Li DJ, Liu QL, Song LB, Li MZ, Zhang RH, Yu XJ, Wang HM, Ernberg I and Zeng YX: Genomic sequence analysis of Epstein-Barr virus strain GD1 from a nasopharyngeal carcinoma patient. J Virol 79: 15323-15330, 2005.

4. Aiyar A, Tyree C and Sugden B: The plasmid replicon of EBV consists of multiple cis-acting elements that facilitate DNA synthesis by the cell and a viral maintenance element. EMBO J 17: 6394-6403, 1998.

5. Sears J, Ujihara M, Wong S, Ott C, Middeldorp J and Aiyar A: The amino terminus of Epstein-Barr Virus (EBV) nuclear antigen 1 contains AT hooks that facilitate the replication and partitioning of latent EBV genomes by tethering them to cellular chromosomes. J Virol 78: 11487-11505, 2004.

6. Wu H, Kapoor P and Frappier L: Separation of the DNA replication, segregation and transcriptional activation functions of Epstein-Barr nuclear antigen 1. J Virol 76: 2480-2490, 2002.

7. Cruickshank J, Shire K, Davidson AR, Edwards AM and Frappier L: Two domains of the epstein-barr virus origin DNAbinding protein, EBNA1, orchestrate sequence-specific DNA binding. J Biol Chem 275: 22273-22277, 2000.

8. Yin Y, Manoury B and Fahraeus R: Self-inhibition of synthesis and antigen presentation by Epstein-Barr virus-encoded EBNA1. Science 301: 1371-1374, 2003.

9. Bhatia K, Raj A, Guitierrez MI, Judde JG, Spangler G, Venkatesh $\mathrm{H}$ and Magrath IT: Variation in the sequence of Epstein Barr virus nuclear antigen 1 in normal peripheral blood lymphocytes and in Burkitt's lymphomas. Oncogene 13: 177-181, 1996.

10. Snudden DK, Smith PR, Lai D, Ng MH and Griffin BE: Alterations in the structure of the EBV nuclear antigen, EBNA1, in epithelial cell tumours. Oncogene 10: 1545-1552, 1995.

11. Gutierrez MI, Raj A, Spangler G, Sharma A, Hussain A, Judde JG, Tsao SW, Yuen PW, Joab I, Magrath IT and Bhatia K: Sequence variations in EBNA-1 may dictate restriction of tissue distribution of Epstein-Barr virus in normal and tumour cells. J Gen Virol 78: 1663-1670, 2000.

12. Mai SJ, Zhang XS, Li DJ, Shen GP, Jiang JH, Zhang RH, Yu XJ, Chen SP and Zeng YX: The character of EBNA1 gene variation in Cantonese and its association with nasopharyngeal carcinoma. Chin Sci Bull 49: 2640-2643, 2004.

13. Zhang XS, Wang HH, Hu LF, Li A, Zhang RH, Mai HQ, Xia JC, Chen LZ and Zeng YX: V-val subtype of Epstein-Barr virus nuclear antigen 1 preferentially exists in biopsies of nasopharyngeal carcinoma. Cancer Lett 211: 11-18, 2004.

14. Wang WY, Chien YC, Jan JS, Chueh CM and Lin JC: Consistent sequence variation of Epstein-Barr virus nuclear antigen 1 in primary tumor and peripheral blood cells of patients with nasopharyngeal carcinoma. Clin Cancer Res 8: 2586-2590, 2002.

15. Mei YP, Zhu XF, Zhou JM, Huang H, Deng R and Zeng YX: siRNA targeting LMP1-induced apoptosis in EBV-positive lymphoma cells is associated with inhibition of telomerase activity and expression. Cancer Lett 18: 1-10, 2005.

16. Chen MR, Zong J and Hayward SD: Delineation of a 16 amino acid sequence that forms a core DNA recognition motif in the Epstein-Barr virus EBNA-1 protein. Virology 205: 486-495, 1994.

17. Sheu LF, Chen A, Meng CL, Ho KC, Lee WH, Leu FJ and Chao CF: Enhanced malignant progression of nasopharyngeal carcinoma cells mediated by the expression of Epstein-Barr nuclear antigen 1 in vivo. J Pathol 180: 243-248, 1996.

18. Wilson JB, Bell JL and Levine AJ: Expression of Epstein-Barr virus nuclear antigen-1 induces B cell neoplasia in transgenic mice. EMBO J 15: 3117-3126, 1996

19. Kang MS, Lu H, Yasui T, Sharpe A, Warren H, CahirMcFarland E, Bronson R, Hung SC and Kieff E: Epstein-Barr virus nuclear antigen 1 does not induce lymphoma in transgenic FVB mice. Proc Natl Acad Sci USA 102: 820-825, 2005. 
20. Kang MS, Hung SC and Kieff E: Epstein-Barr virus nuclear antigen 1 activates transcription from episomal but not integrated DNA and does not alter lymphocyte growth. Proc Natl Acad Sci USA 98: 15233-15238, 2001.

21. Sandvej K, Zhou XG and Hamilton-Dutoit S: EBNA-1 sequence variation in Danish and Chinese EBV-associated tumours: evidence for geographical polymorphism but not for tumourspecific subtype restriction. J Pathol 191: 127-131, 2000.

22. Gahn TA and Sugden B: An EBNA-1-dependent enhancer acts from a distance of 10 kilobase pairs to increase expression of the Epstein-Barr virus LMP gene. J Virol 69: 2633-2636, 1995.

23. Srinivas SK and Sixbey JW: Epstein-Barr virus induction of recombinase-activating genes RAG1 and RAG2. J Virol 69: 8155-8158, 1995.
24. Kube D, Vockerodt M, Weber O, Hell K, Wolf J, Haier B, Grasser FA, Muller-Lantzsch N, Kieff E, Diehl V and Tesch H: Expression of Epstein-Barr virus nuclear antigen 1 is associated with enhanced expression of CD25 in the Hodgkin cell line L428. J Virol 73: 1630-1636, 1999.

25. Kuhn-Hallek I, Sage DR, Stein L, Groelle H and Fingeroth JD: Expression of recombination activating genes (RAG-1 and RAG-2) in Epstein-Barr virus-bearing B cells. Blood 85: 1289-1299, 1995.

26. Tsimbouri P, Drotar ME, Coy JL and Wilson JB: bcl-xL and RAG genes are induced and the response to IL-2 enhanced in EmuEBNA-1 transgenic mouse lymphocytes. Oncogene 21: 5182-5187, 2002 . 